\title{
Brain Mapping Project: Clinical Aspects and Role of Neuroradiology
}

\section{ABSTRACT}

SUMMARY: As experts in the realm of applying brain imaging technology for meaningful information on an individual patient's medical care, neuroradiologists can provide a physiologic, pathophysiologic, and clinical context for the advances achieved from brain-mapping. Precise identification of the abnormalities involved in brain conditions resulting from a multidisciplinary effort including neuroradiology will help to further define the nature of the pathology, evaluate the results of therapy, and improve health care.

B rain mapping generally involves the study of the anatomy and function of the brain and spinal cord with imaging. It incorporates many approaches, ranging from neuroimaging, neurosurgical intraoperative procedures (microscopic, endoscopic, and multitechnique imaging), immunohistochemistry, molecular and ontogenetic studies, stem cell and cellular biology, engineering disciplines (material, electrical, and biomedical), and neurophysiology to nanotechnology. ${ }^{1}$ The synergy of these disciplines will provide improved understanding of the human brain as viewed from each of these complementary perspectives.

The mechanisms by which the brain functions and neurons are interconnected are of paramount importance and usefulness both for clinical applications and as inspiration for novel technologies. To date, advances in brain mapping have considerably aided in understanding, diagnosing, and treating various neurologic conditions. For example, mapping the location of important brain regions such as language and motor function by using neuroimaging can help neurosurgeons accurately pinpoint important and diseased regions of the brain to minimize resection of vital, functioning regions during neurosurgery. Knowledge of how the brain is wired and can become rewired is still evolving and can be of use in understanding how it functions and recovers following disease, injury, or surgery.

However, mapping brain function only is not enough; we need to define the normal and abnormal functions and include the related or potential therapeutic aspects. The clinical goal of brain mapping is to develop and use methods for the understanding,

From the Department of Radiology, George Washington University Medical Center, Washington, DC.

Please address correspondence to L.M. Levy, MD, PhD, Professor of Radiology and Director of Neuroradiology, George Washington University Medical Center, Washington, DC 20037; e-mail: llevy@mfa.gwu.edu

http://dx.doi.org/10.3174/ajnr.A3587 diagnosis, and clinical management of normal brain function and neurologic conditions, with studies extending from the macro to the micro and nano levels.

Alterations of brain function levels have already been related to the pathophysiology of a number of neurologic conditions, including tumors, stroke, dementias, trauma, neuroplasticity, movement disorders, epilepsy, demyelinating disease, neurometabolic diseases, migraine, drug abuse, and neuroendocrine diseases. Conventional anatomic imaging methods such as CT or MR imaging have not proved sufficient in the evaluation of these disorders. The corollary goals of this project include the development, improvement, and validation of novel treatment and imaging techniques.

Brain mapping information can provide clinicians with a powerful tool to evaluate and serially follow diseases. Measurements of certain cerebral markers, such as the neurotransmitters that mediate brain function, can permit quantitative assessment of disease progression with time and drug-mediated effects. These markers can also be used to detect specific diseases such as Alzheimer disease and to evaluate disease progression and treatment response. They may also allow the development of entirely novel approaches to treatment that are rational, on the basis of the findings of these studies. Early detection of these neurologic conditions would provide a greater chance for appropriate and timely treatment of patients and for delivery of better health care. These methods should encompass new techniques for the detection, mapping, and treatment of diseases. Recent advances have the potential to dramatically enhance the detection of neurologic diseases with targeted contrast agents, for example, and to deliver focused therapies to the central nervous system. These developments will greatly impact diagnostics and therapeutics across many medical specialties in the coming age of personalized and tar- 
geted medicine. These effects will likely be seen in many areas, including stem cell therapy, immunoimaging and immunotherapy, and will enhance pre- and intraoperative brain tumor characterization, nanoneurosurgery, and targeted therapeutic payload delivery to the nervous system.

A brain mapping project or roadmap should include the development of data bases and methods to evaluate the following:

1) How the brain is wired or connected.

2) How the brain functions and processes information.

3) How different neurologic conditions are related to connectivity patterns.

4) How neurologic conditions are related to brain function patterns.

5) How brain structure and function are related to various types of therapies.

6) How to detect brain disorders.

7) How to evaluate disease progression and results of treatment.

Determination of brain connectivity and functional profiles will enable us to further evaluate changes associated with pathology or treatment and to follow progression of disease or response to therapy.

For this endeavor, a systematic organization of effective teams, involving a wide spectrum of specialties, including neuroscience, neuropsychology, neurology, neurosurgery, engineering (including material, biomedical, electrical, and aero- nautical), and neuroradiology, will be needed. A systematic team approach and the sharing of information and experience will lead to a more efficient use of our collaboration across the biologic and physical sciences and the funding of resources. As experts in the realm of applying brain imaging technology for meaningful information on an individual patient's medical care, neuroradiologists can provide a physiologic, pathophysiologic, and clinical context for the advances achieved from brain mapping. Precise identification of the abnormalities involved in brain conditions resulting from a multidisciplinary effort, including neuroradiology, will help to further define the nature of the pathology, evaluate the results of therapy, and improve health care. In addition, the technologies employed and the findings of these studies may have applications beyond the clinical realm.

\section{ACKNOWLEDGMENTS}

I thank the members of the Society for Brain Mapping and Therapeutics (especially Dr B. Kateb) for their effort in building multidisciplinary groups to complement the work already done by other societies and research institutes.

\section{REFERENCE}

1. Kateb B, Heiss JD, eds. The Textbook of Nanoscience and Nanoneurosurgery. CRC Press, Taylor \& Francis Group LLC; 2013 